Arq. Bras. Med. Vet. Zootec., v.66, n.3, p.787-796, 2014

\title{
Anestesia epidural na cirurgia descompressiva lombossacral de cães
}

\author{
[Epidural anesthesia in the lumbosacral decompressive surgery in dogs] \\ E.A. Tudury ${ }^{1}$, T.H.T. Fernandes ${ }^{2 *}$, M.L. Figueiredo ${ }^{2}$, B.M. Araújo ${ }^{2}$, M.A. Bonelli, \\ A.C. Silva ${ }^{2}$, C.R.O. Santos ${ }^{3}$ \\ ${ }^{1}$ Universidade Federal Rural de Pernambuco -UFRPE - Recife, PE \\ ${ }^{2}$ Alunos de pós-graduação - Universidade Federal Rural de Pernambuco - UFRPE - Recife, PE \\ ${ }^{3}$ Aluna de graduação - Universidade Federal Rural de Pernambuco -UFRPE - Recife, PE
}

\begin{abstract}
RESUMO
Objetivou-se avaliar viabilidade, eficácia, vantagens e desvantagens da anestesia epidural lombossacral, junto à anestesia geral inalatória, em cirurgias de descompressão da cauda equina, tendo em vista que essas cirurgias são procedimentos longos e dolorosos. Para isso, foram utilizados 11 cães adultos com sinais clínicos de síndrome da cauda equina, que foram submetidos à anestesia geral inalatória e deixados no estágio anestésico mais superficial. Depois, realizou-se a anestesia epidural em seis dos 11 pacientes, por punção espinhal em $\mathrm{L}_{7}-\mathrm{S}_{1}$, com bupivacaína a 0,5\%. Os parâmetros fisiológicos (cardíacos, vasculares, respiratórios, temperatura corporal e glicemia) foram aferidos antes da medicação préanestésica, 10 minutos após esta, 30 minutos depois da epidural, depois da laminectomia, assim como após 60 minutos e 90 minutos da epidural, tanto no grupo com epidural como naquele sem esta (controle). Os animais que possuíam bloqueio epidural apresentaram redução significativa no consumo de anestésico inalatório e no tempo de extubação, não apresentando déficits neurológicos causados pela anestesia epidural, quando comparados com o grupo-controle. Conclui-se que a técnica de anestesia epidural é eficiente e vantajosa na realização de cirurgias descompressivas lombossacrais, pois proporciona menor risco anestésico para o animal.
\end{abstract}

Palavras-chave: cauda equina, bloqueio anestésico, analgesia

\begin{abstract}
The objective of this study was to evaluate the feasibility, effectiveness, advantages and disadvantages of lumbosacral epidural anesthesia with inhalational anesthesia in surgical decompression of the cauda equina, since this surgery has a lengthy and painful recovery from anesthesia. For this, 11 adult dogs which showed clinical signs of cauda equina syndrome were used. After the epidural, anesthesia in six of 11 patients, spinal tap in $L_{7}-S_{1}$, with bupivacaine $0.5 \%$ anesthetic was used. Physiological parameters (heart, vascular, respiratory and body temperature) were measured before, 10 minutes after, and 30 minutes after the epidural, after the laminectomia and 60 minutes and 90 minutes after the epidural, both in the epidural group and the control group. Animals that had ep showed a significant reduction in consumption of inhaled anesthetic and time of extubation. There were no neurological deficits caused by epidural anesthesia compared with the group without epidural anesthesia. It was concluded that the epidural anesthesia technique is effective in lumbosacral decompression surgeries, leading to a lower risk for the anesthetized animal.
\end{abstract}

Keywords: cauda equina, anesthesia, analgesia

Recebido em 2 de março de 2012

Aceito em 6 de setembro de 2013

*Autor para correspondência (corresponding author)

E-mail: thaizavet@gmail.com 


\section{INTRODUÇÃO}

A síndrome da cauda equina é um conjunto de sinais neurológicos decorrentes da compressão das raízes nervosas situadas no canal espinhal lombossacral (Seim III, 2005). É provocada por afecções como estenose congênita, doença do disco intervertebral, trauma, discoespondilite, isquemia (Tarvin e Prata, 1980), neoplasia, luxação, osteomielite vertebral, êmbolo fibrocartilaginoso e osteocondrose sacral (Seim III, 2005). Em estudo no Hospital Veterinário da Universidade Federal Rural de Pernambuco (UFRPE), de 254 cães com problemas neurológicos variados, $11 \%$ sofriam de estenose lombossacral, com consequente compressão da cauda equina (Araujo, 2010).

A cirurgia na região lombossacral estimula e irrita os nervos: ciático, responsável pela percepção sensorial na superfície lateral, cranial e plantar do membro pélvico; pudendo, com função sensorial no períneo, ânus e genitais; pélvico e sacro, que transmitem sensações viscerais advindas do canal pélvico; e finalmente os nervos caudais, com função sensorial na cauda (Sjostrom, 2007). Esses nervos normalmente têm sua condução neural bloqueada ao se efetuar uma anestesia epidural (Massone, 2003).

Apesar de os anestésicos gerais promoverem imobilidade, inconsciência e amnésia, eles não bloqueiam as aferências do sistema nervoso sensitivo ao corno dorsal da medula espinhal e a sensibilização central, pois são desprovidos de efeito analgésico (Katz, 2001). Durante o procedimento cirúrgico, frente ao estímulo doloroso constante, grupos de neurônios sofrem alterações na modulação e nocicepção, provocando grande irritabilidade e intensa excitação (Dobromyslskyj et al., 2001). O uso exclusivo de anestésicos gerais, com bloqueio incompleto das vias sensitivas, é responsável pelo aumento da intensidade da dor e do consumo de analgésicos no período pósoperatório (Oliveira et al., 2004), o que acarreta alterações fisiológicas e hemodinâmicas prejudiciais ao paciente (Fantoni e Mastrocinque, 2010).

A anestesia epidural, apesar de ser uma técnica antiga, vem sendo bastante utilizada por possuir vantagens como possibilitar o emprego de doses pequenas de fármacos e anestésicos gerais, com poucos efeitos colaterais, e promover analgesia trans e pós-operatória (McMurphy, 1993). Quando utilizada com anestésicos locais, gera bloqueio medular e de nervos espinhais (Collins, 1993), pois esses fármacos possuem afinidades pelo tecido nervoso, interrompendo a condução elétrica nos neurônios (Pascoe, 1997; Massone, 2003). Entretanto, em cirurgia de descompressão da cauda equina, não existem citações na literatura pesquisada da utilização dessa técnica de bloqueio regional.

As vantagens dessa anestesia são um menor índice de mortalidade pelo fato de a necessidade da anestesia geral ser menos profunda e, assim, gerar redução do estresse operatório por meio da eliminação dos estímulos dolorosos aferentes do local da cirurgia (Brown e Wedell, 1993).

Os bloqueios nervosos, com anestésicos locais, são capazes de aliviar e neutralizar a sensação dolorosa severa. Na laminectomia, por ser geradora de dor severa, devem-se planejar estratégias analgésicas adequadas no pré, trans e pós-operatório (Mich e Hellyer, 2008).

Uma vez que a literatura não descreve a realização da anestesia epidural em cirurgias descompressivas lombossacrais, objetivou-se avaliar viabilidade, eficácia, vantagens e desvantagens da anestesia epidural lombossacral, junto à anestesia geral inalatória, em cirurgias de descompressão da cauda equina, tendo em vista que essa cirurgia é um procedimento longo e doloroso.

\section{MATERIAL E MÉTODOS}

Após aprovação do projeto pela Comissão de Ética para Uso de Animais (CEUA) da Universidade Federal Rural de Pernambuco (UFRPE), com devida liberação de licença sob o número 001/2011, foram utilizados 11 cães adultos do atendimento de rotina do Hospital Veterinário da UFRPE, sem distinção de sexo, que apresentaram síndrome da cauda equina diagnosticada por meio de exames neurológicos e radiográficos (simples e contrastados).

Realizou-se anamnese detalhada visando obter informações e histórico clínico de cada paciente. O exame físico dos animais constou de avaliação clínica geral e neurológica. Foram incluídos, na pesquisa, os animais que se encontravam 
clinicamente estáveis para anestesia cirúrgica, com percepção normal a estímulos de dor, efetuados manualmente nos membros posteriores e na região a ser operada, e cujos tutores concordaram com a realização do procedimento.

Todos os pacientes foram submetidos à anestesia geral inalatória e deixados no estágio anestésico mais superficial, estágio III, em plano 1 para o grupo com bloqueio e em plano 2 para o grupo sem bloqueio (avaliado pela escala de Guedel), o qual permitisse realizar a cirurgia sem que os animais sentissem dor, para execução das comparações da pesquisa.

Os 11 pacientes foram submetidos a jejum hídrico de duas horas, sólido de 12 horas e uma pré-medicação anestésica (MPA) com os fármacos acepromazina $0,2 \%$ a $0,05 \mathrm{mg} / \mathrm{kg}$ por via intravenosa (IV), em associação na mesma seringa com diazepam $0,5 \%$ a $0,3 \mathrm{mg} / \mathrm{kg}$ e cloridrato de tramadol $(2 \mathrm{mg} / \mathrm{kg}$ intramuscularIM). Durante o trans- operatório, os animais receberam fluido de $\mathrm{NaCl}$ 0,9\%. Decorridos 15 minutos, a indução foi realizada com propofol $(4 \mathrm{mg} / \mathrm{kg}, \mathrm{IV})$, seguida de intubação endotraqueal, e a manutenção inalatória com isoflurano (controle em $\mathrm{mL} / \mathrm{kg}$ ) diluído em oxigênio 100\%, com fluxo controlado por meio de vaporizador universal.

A anestesia epidural foi realizada em seis dos 11 pacientes, por punção espinhal em $\mathrm{L}_{7}-\mathrm{S}_{1}$, com bupivacaína a $0,5 \%$ sem vaso constritor $(0,25 \mathrm{~mL} / \mathrm{kg})$, aplicada com agulha de cateter intravenoso de número de acordo com o porte do animal e guiada por eletroneuroestimulador. Para a certificação do bloqueio, foram realizados os testes dos reflexos patelares e flexores, tônus muscular e dermátomos cutâneos dos membros posteriores, com os animais no plano 1 do estágio III.

Para o monitoramento transanestésico, foi utilizado aparelho multiparamétrico em derivação II para acompanhamento do traçado eletrocardiográfico, do ritmo cardíaco e da frequência cardíaca (FC), esta registrada em batimentos por minutos (bpm), e a saturação de oxi-hemoglobina (Sp02) por leitura no monitor $(\%)$.

A frequência respiratória (FR), em movimentos por minuto (mpm), foi aferida pelo monitor acima citado e por observação do balão do aparelho de anestesia inalatória.

Foi realizada leitura da pressão arterial sistólica (PAS), em mmHg, mediante Doppler vascular, com manguito conectado ao membro torácico direito.

Também foi verificada a temperatura retal $(\mathrm{T})$, em graus Celsius $\left({ }^{\circ} \mathrm{C}\right)$, com termômetro conectado ao monitor multiparamétrico.

O consumo de anestésico inalatório (ISOmL) foi mensurado em mililitros $(\mathrm{mL})$ por hora por quilo, calculado pela diferença observada na escala graduada entre o volume colocado no início e o que restou no vaporizador ao final do procedimento. O tempo de extubação (Tex), em minutos, foi aferido entre a interrupção do fornecimento do isoflurano e a tentativa de expulsão espontânea da sonda endotraqueal. Também foi registrado o tempo total da cirurgia.

Para verificação de sensação ou não de dor, foi coletada amostra sanguínea para dosagem de glicose antes da cirurgia, ao início do procedimento anestésico e ao término da cirurgia (último ponto cutâneo). As amostras sanguíneas foram analisadas por glicosímetro portátil Accu$\mathrm{Chek}^{\circledR}$. Foram verificados a vocalização e o retorno do animal da anestesia.

Todos esses parâmetros fisiológicos (cardíacos, vasculares, respiratórios e temperatura corporal) foram aferidos antes da MPA (M0), 10 minutos após (M1), 30 minutos depois de concluído o procedimento de anestesia epidural (M2), depois da laminectomia (M3), assim como 60 minutos (M4) e 90 minutos (M5) após a epidural, tanto no grupo com epidural como naquele sem ela, para posterior comparação estatística.

A avaliação do bloqueio sensitivo foi feita por pinçamento dos dermátomos acima das vértebras, da cauda, dos interdígitos, do ânus e da vulva ou do bulbo do pênis dos animais, e o bloqueio motor foi feito pela perda do tônus muscular do membro pélvico e da cauda. Esses bloqueios também foram verificados pela avaliação dos arcos reflexos patelar e flexor.

Após a cirurgia, os pacientes receberam antibioticoterapia oral (30 mg/kg de cefalexina) a cada oito horas, durante sete dias consecutivos. 
Para analgesia, foi utilizado, por via oral, meloxicam $(0,1 \mathrm{mg} / \mathrm{kg}$, a cada 24 horas, durante sete dias) e tramadol $(2 \mathrm{mg} / \mathrm{kg}$, a cada oito horas, por cinco dias).

As variáveis analisadas foram temperatura $\left({ }^{\circ} \mathrm{C}\right)$, frequência cardíaca (bpm), frequência respiratória (mpm), saturação de oxigênio (\%), pressão arterial sistólica $(\mathrm{mmHg})$, consumo de anestésico isoflurano por hora e por peso $(\mathrm{mL} / \mathrm{h} / \mathrm{kg}$ ), tempo de extubação (minutos) e níveis de glicose sérica (MG/gL). Todas as variáveis foram comparadas entre os animais que receberam anestesia epidural e os animais que não receberam a citada anestesia.

Os dados foram analisados por meio de tabelas, gráficos, medidas de tendência central (médias), medidas de dispersão (desvio-padrão) e testes de hipóteses. Para as variáveis temperatura, frequência cardíaca, frequência respiratória, saturação de oxigênio, pressão arterial sistólica, consumo de anestésico isoflurano por hora e por peso, e tempo de extubação, foi utilizado o teste não paramétrico para amostras independentes de Mann-Whitney; e para a variável glicose, foi utilizado o teste paramétrico de t pareado.
As análises estatísticas foram realizadas mediante o uso do software estatístico GraphPad Instat ${ }^{\circ}$, versão 3.0. O nível de significância adotado foi de 5\% para todos os testes realizados.

\section{RESULTADOS}

Dos animais do experimento, 10 eram machos (10/11) e um fêmea (1/11) e de raças variadas: sem raça definida (3/11), Poodle (2/11), Pastor alemão (2/11), Rottweiler (1/11), Golden Retriever (1/11), Rhodesian Ridgeback (1/11) e Yorkshire (1/11). A idade deles situou-se entre nove meses e nove anos, com média de quatro anos.

As afecções que causaram a compressão lombossacra foram hérnia de disco entre $\mathrm{L}_{7} \mathrm{~S}_{1}$ (7/11), fratura de $L_{7}(2 / 11)$ e luxação de $L_{7}$ $(2 / 11)$.

As médias e os desvios-padrão dos valores de T, FC, FR, SpO2 e PAS dos grupos com anestesia epidural e sem anestesia epidural, em vários momentos, estão representados na Tab. 1.

Tabela 1. Valores médios e desvios-padrão da temperatura $\left({ }^{\circ} \mathrm{C}\right)$, frequência cardíaca (bpm), frequência respiratória (mpm), saturação de oxigênio $(\%)$ e pressão arterial sistólica $(\mathrm{mmHg})$ dos grupos com epidural (CE) e sem epidural (SE) nos seis momentos de avaliação

\begin{tabular}{|c|c|c|c|c|c|c|c|}
\hline Parâmetros & Grupo & M0 & M1 & M2 & M3 & M4 & M5 \\
\hline Temperatura & $\mathrm{CE}$ & $38,7 \pm 0,603$ & $37,74 \pm 0,937$ & $37,78 \pm 0,682$ & $37,70 \pm 0,565$ & $37,61 \pm 0,370$ & $37,72 \pm 0,402$ \\
\hline$\left({ }^{\circ} \mathrm{C}\right)$ & SE & $38,66 \pm 0,181$ & $38,14 \pm 0,472$ & $38,36 \pm 0,763$ & $38,18 \pm 0,816$ & $38,28 \pm 0,878$ & $37,88 \pm 1,068$ \\
\hline FC & $\mathrm{CE}$ & $99,66 \pm 19,586$ & $84,00 \pm 27,865$ & $90,33 \pm 20,490$ & $92,33 \pm 19,612$ & $94,16 \pm 28,512$ & $99,33 \pm 27,383$ \\
\hline & SE & $117,80 \pm 49,479$ & $126,40 \pm 23,765$ & $134,80 \pm 24,097$ & $131,60 \pm 15,646$ & $123,0 \pm 21,610$ & $132,40 \pm 9,633$ \\
\hline $\begin{array}{l}\text { FR } \\
\text { (mpm) }\end{array}$ & $\mathrm{CE}$ & $33,0 \pm 3,521$ & $19,20 \pm 7,155$ & $16,66 \pm 5,316$ & $16,0 \pm 5,059$ & $17,33 \pm 3,265$ & $17,33 \pm 8,262$ \\
\hline & SE & $20,80 \pm 7,155$ & $19,20 \pm 4,381$ & $16,80 \pm 1,788$ & $15,20 \pm 4,381$ & $16,80 \pm 1,788$ & $19,20 \pm 4,381$ \\
\hline $\begin{array}{l}\mathrm{SPO} 2 \\
(\%)\end{array}$ & $\mathrm{CE}$ & $100 \pm 0$ & $95,40 \pm 5,554$ & $94,33 \pm 5,715$ & $93,83 \pm 6,765$ & $95,33 \pm 2,804$ & $95,2 \pm 3,271$ \\
\hline PAS (mmHg) & $\begin{array}{l}\text { SE } \\
\text { CE } \\
\text { SE }\end{array}$ & $\begin{array}{c}97,4 \pm 4,219 \\
79,66 \pm 5,715 \\
112,0 \pm 36,331\end{array}$ & $\begin{array}{c}97,8 \pm 1,095 \\
81,66 \pm 7,527 \\
114,00 \pm 29,664\end{array}$ & $\begin{array}{c}96,60 \pm 2,073 \\
88,66 \pm 9,933 \\
108,0 \pm 34,205\end{array}$ & $\begin{array}{c}97,6 \pm 1,516 \\
83,33 \pm 8,164 \\
114,00 \pm 39,115\end{array}$ & $\begin{array}{c}96,60 \pm 3,049 \\
84,16 \pm 8,010 \\
112,0 \pm 29,495\end{array}$ & $\begin{array}{c}97,40 \pm 2,190 \\
78,33 \pm 4,082 \\
117,0 \pm 26,832\end{array}$ \\
\hline
\end{tabular}

Em relação à frequência cardíaca, não houve diferença significativa $(p=0,5368)$ entre os grupos com anestesia epidural e sem anestesia epidural, em qualquer momento, apesar de haver menor frequência no grupo com epidural (Fig. 1a).

Quanto às médias dos valores obtidos da saturação periférica de hemoglobina ( $\mathrm{SpO} 2)$, não foi observada diferença estatisticamente significativa $(p=0,4637)$ entre os grupos (com epidural e sem epidural), como mostrado na Fig. 1b.

Ao se analisarem os dados referentes à variação da PAS, não foi observada diferença estatisticamente significativa $(\mathrm{p}=0,0815)$ entre os grupos (com epidural e sem epidural), como mostrado na Fig. 1c. 


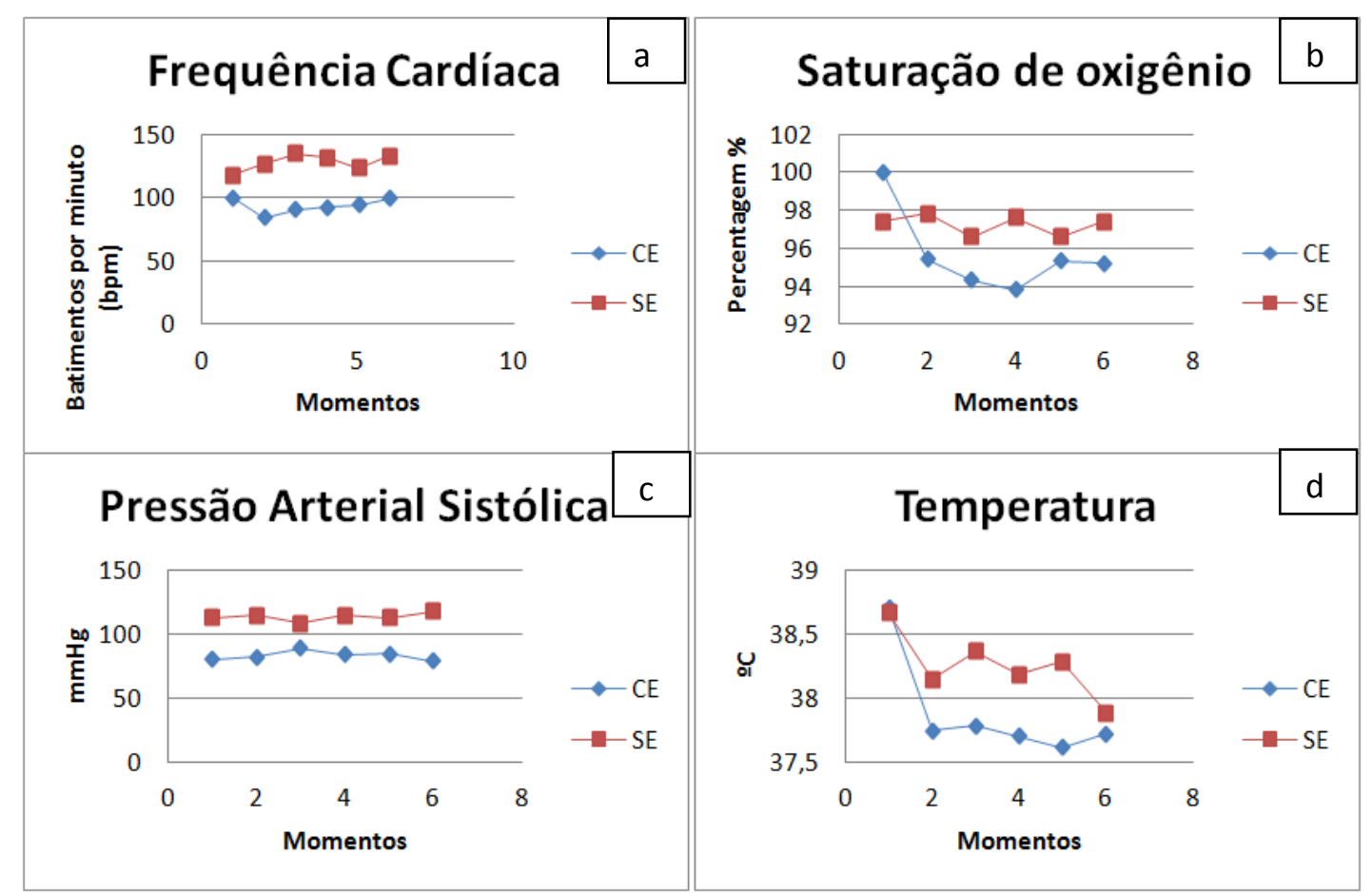

Figura 1. Representação gráfica da distribuição das médias de frequência cardíaca (a), de saturação de oxigênio (b), de pressão arterial sistólica (c) e de temperatura (d) dos grupos com (CE) e sem (SE) epidural nos seis momentos de avaliação.

Com relação à variação da temperatura, não foi observada diferença estatisticamente significativa $(\mathrm{p}=0,5835)$ entre os grupos (com epidural e sem epidural), como representado na Fig. 1d, apesar de o grupo com extremos valores sempre exercer influência em todos os tempos de avaliação.

Quanto à variação da FR, não foi observada diferença estatisticamente significativa $(\mathrm{p}=0,0444)$ entre os grupos (com epidural e sem epidural), exceto no momento M0.

Já no tocante à variação do consumo de isoflurano em relação ao tempo de cirurgia e ao peso do animal, foi observada diferença estatisticamente significativa $(\mathrm{p}=0,0446)$ entre os grupos, o que resultou em um consumo aproximadamente 10 vezes menor de anestésico nos animais com bloqueio epidural (Fig. 2).
Relativamente ao tempo de extubação, foi observada diferença altamente significativa $(\mathrm{p}=0,0043)$ entre os grupos (com epidural e sem epidural), com um tempo sete vezes menor no grupo com epidural (Fig. 3).

Considerando-se a variação dos níveis de glicose antes e após o procedimento cirúrgico nos animais em que foi realizada anestesia epidural, não foi observada diferença estatisticamente significativa $(\mathrm{p}=0,0173)$, enquanto no grupo sem anestesia epidural, os níveis de glicose antes e após tiveram nos seus valores diferença estatisticamente significativa $(\mathrm{p}=0,0098)$. Por animal, ocorreu um aumento da glicose tanto no grupo sem anestesia epidural como naquele com bloqueio, o que é mostrado nas Fig. 4A e 4B. 


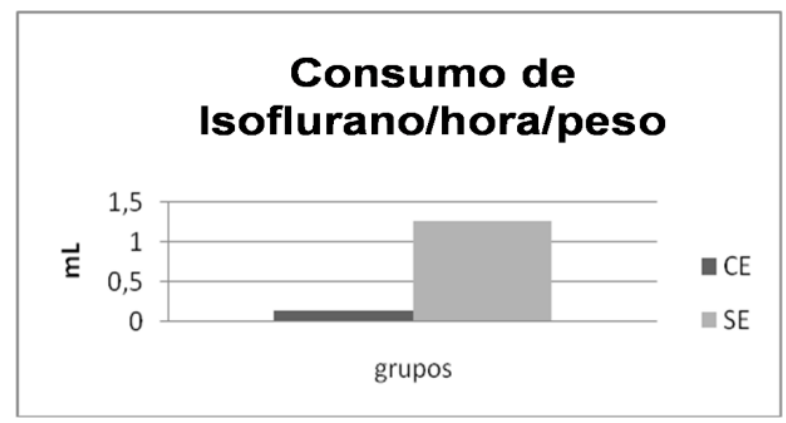

Figura 2. Representação gráfica das médias do consumo de isoflurano em $\mathrm{mL}$ em relação ao tempo de cirurgia (h) e ao peso do animal (kg) nos grupos com (CE) e sem (SE) bloqueio epidural.

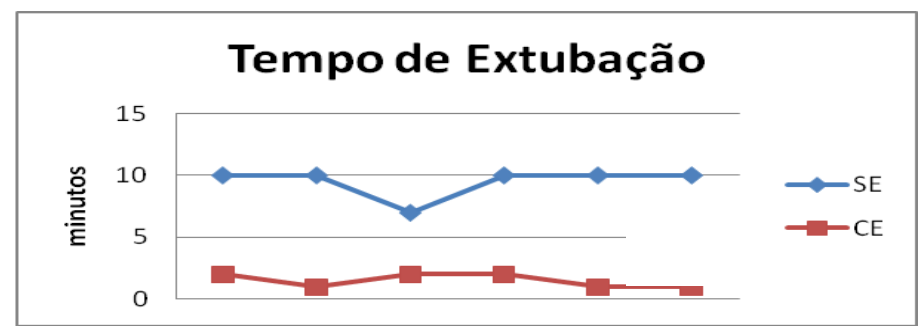

Figura 3. Tempo de extubação, em minutos, dos animais do experimento nos grupos com (CE) e sem (SE) epidural.

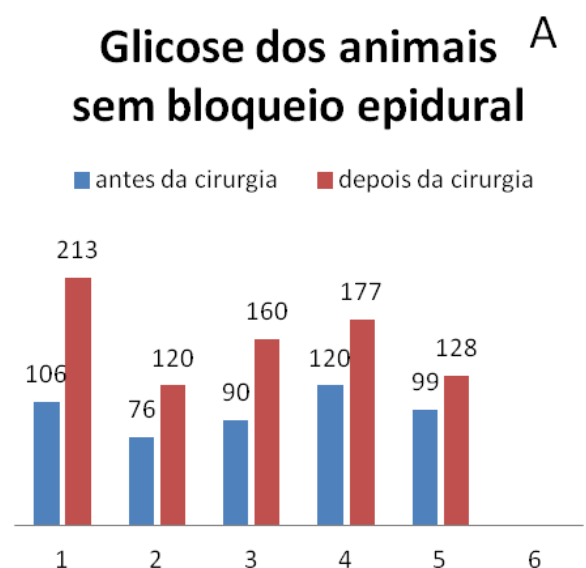

\section{Glicose dos animais com bloqueio epidural}

antes da cirugia $\quad$ depois da cirurgia

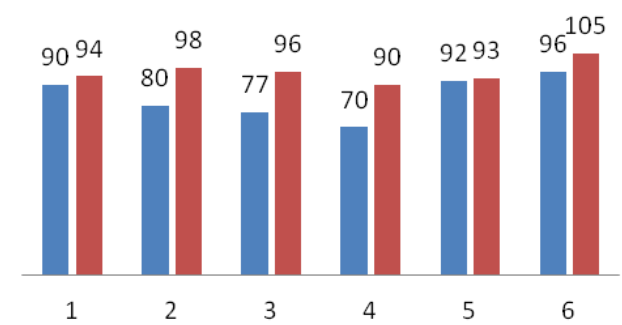

Figura 4. Representação gráfica da glicose $\left(\mathrm{em} \mathrm{mg.dL} \mathrm{L}^{-1}\right)$ dos animais no grupo sem bloqueio epidural (A) e com bloqueio (B), antes e depois do procedimento cirúrgico.

\section{DISCUSSÃO}

A anestesia epidural foi tema desta pesquisa não só por constituir uma técnica vantajosa, sendo importante recurso no trans e pós-operatório, ao auxiliar o relaxamento muscular e a analgesia pós-operatória e proporcionar uma recuperação de melhor qualidade (Carvalho e Luna, 2007), mas também por não haver relatos na literatura de sua realização em cirurgias descompressivas lombossacrais. A anestesia mencionada para esses procedimentos é a anestesia geral inalatória, e não sua associação (Palmer e Chambers, 1991; Bojrab, 1996; Lagedo et al., 1999; Risio et al., 2000; Seim III, 2005; Sharp e Wheeler, 2005; Sjostrom, 2007; Cariou et al., 2008; Beckmann, 2010).

A adoção de diazepam e tramadol como prémedicação em todos os animais nos dois grupos 
teve como objetivo potencializar o efeito dos outros anestésicos, reduzir a dose de propofol e causar analgesia (Wilder-Smith et al., 2001), a fim de minimizar as variações e a resposta entre os grupos antes do bloqueio. O diazepam é destituído de propriedades analgésicas, da mesma forma que o propofol em doses baixas (Thumon et al., 1996), não interferindo sobremaneira na resposta nociceptiva dos cães (Sebel e Lowdon, 1989).

Foi necessário que os animais fossem anestesiados anteriormente à anestesia epidural, pois, para a realização dessa técnica, é preciso que o paciente esteja imóvel para que não haja acidentes com a agulha e lesões neurológicas, segundo Pinheiro et al. (1996).

Segundo Palmer e Chambers (1991), Sjostrom (2007) e Cariou et al. (2008), a síndrome da cauda equina comumente afeta cães de meiaidade, de raças de médio a grande porte, particularmente machos, porém, em relação à idade, neste trabalho, 54,5\% dos cães tinham idade inferior a quatro anos.

Em todos os animais do grupo da anestesia epidural houve bloqueio sensitivo por mais de cinco horas, corroborando Skarda (1996), que relata obtenção de anestesia cirúrgica com bupivacaína $0,5 \%$ por quatro a seis horas. Assim, a recuperação anestésica ocorreu de forma tranquila e silenciosa. Já no grupo sem anestesia epidural, quatro dos cinco animais (80\%) retornaram de maneira agitada e com vocalização indicativa de dor, sendo necessárias doses de morfina no pós-cirúrgico imediato, o que confirma que o tramadol não é suficiente para aliviar dores severas (Mich e Hellyer, 2008) e que, durante a cirurgia, não interferia nos resultados.

Utilizou-se tramadol na MPA por via intravenosa e não por via epidural para padronizar os grupos e não haver influência deste fármaco no experimento, pois só funciona para aliviar dor discreta e moderada (Mich e Hellyer, 2008). O mesmo raciocínio foi empregado com relação à opção de não usar vasoconstritor associado ao anestésico local.

Em investigação das médias de frequência cardíaca, temperatura retal, saturação de oxigênio e pressão arterial, em cães submetidos à técnica epidural, não foram encontradas alterações significativas nas variáveis estudadas quando relacionadas às do grupo sem bloqueio anestésico, o que está em concordância com Nunes et al. (1993), que descreveram os efeitos cardiorrespiratórios da anestesia epidural em cães.

Ao se avaliarem as alterações do sistema respiratório, observou-se que os grupos se comportaram de maneira semelhante no tocante aos valores de frequência respiratória e que houve diferença no primeiro momento, justificado pelo fato de o grupo com anestesia epidural apresentar animais mais agitados.

Em nenhum dos grupos avaliados foi observada diminuição da saturação de oxigênio durante o procedimento cirúrgico, o que seguramente decorreu da tentativa de manter os animais no plano anestésico mais superficial que possibilitasse a cirurgia, pois esses valores caem em anestesias muito profundas, com diminuição da frequência e profundidade respiratórias (Fantoni e Cortopassi, 2010).

Em relação aos procedimentos cirúrgicos, sabese que fatores estressantes, como, por exemplo, a dor, podem levar à liberação de hormônios, que, entre outros, promovem a gliconeogênese e a glicogenólise, provocando aumento nos valores de glicemia, tanto no animal como no homem (Nogueira et al., 2003). Como as neurocirurgias lombossacrais são geradoras de dor severa (Lauretti et al., 2007), optou-se por aferição dos valores glicêmicos no pré e pós-cirúrgico como parâmetro de sofrimento do animal, o que mostrou que a não realização do bloqueio levou a um aumento da glicose sérica nesse grupo. No grupo-controle (sem anestesia epidural), houve um pequeno aumento da glicose, mas dentro dos padrões, justificado pelos fármacos utilizados no procedimento anestésico.

A maioria dos animais apresentava hérnia de disco, enquanto que em menor número havia luxação ou fratura. Estas lesões compressivas não afetaram a difusão do anestésico local no espaço epidural, já que houve correta anestesia epidural. Além disso, a técnica de bloqueio não interferiu no procedimento cirúrgico de descompressão, discordando de Hebl e colaboradores (2006), quando afirmam que a anestesia epidural pode agravar a lesão 
neurológica encontrada nas hérnias e na compressão lombossacral.

Entre as vantagens da técnica de anestesia epidural, pode-se citar: baixo custo, excelente relaxamento muscular, efeito sistêmico mínimo e retorno rápido da anestesia geral, constatados neste experimento ao se compararem os tempos de extubação entre os dois grupos, corroborando Blass e Shire (1987) e Nunes et al. (1993). Porém, o presente estudo diverge de conclusões de Blass e Shire (1987), que relatam como desvantagens as reações tóxicas, a dificuldade respiratória e a não possibilidade de realização dessa técnica em traumas (Intelizano et al., 2002), o que foi conseguido no cão com fratura e deslocamento cranioventral de $\mathrm{L}_{7}$, com bloqueio satisfatório.

Como resultado do bloqueio epidural aqui observado, pode-se citar a redução do risco de depressão anestésica, que seria uma vantagem, principalmente, para pacientes em estado grave e para idosos (Carvalho e Luna, 2007).

Em animais obesos com fraturas e luxações de vértebras, pela difícil localização do espaço epidural, foi utilizado um eletroestimulador como guia neste experimento para a confirmação do bloqueio epidural pelas respostas musculares axiais aos estímulos nervosos, já que o sucesso da anestesia epidural depende da localização correta do espaço epidural (Carvalho e Luna, 2007).

O tempo de extubação médio no grupo sem bloqueio foi de 10 minutos, o que está de acordo com estudos de Polis et al. (2001). No grupo com epidural, entretanto, foi, aproximadamente, sete vezes menor (1,5 minuto), podendo ser assim mais rápida a recuperação anestésica dos animais submetidos à cirurgia lombossacral, pois a manutenção em plano anestésico mais profundo pode trazer prejuízos ao paciente, como hipotermia, hipotensão arterial e depressão respiratória (Fantoni e Cortopassi, 2010).

No presente estudo, a quantidade de isoflurano consumido $(\mathrm{mL})$ em relação ao tempo de cirurgia (h) e ao peso do animal ( $\mathrm{kg}$ ) foi também muito menor no grupo com bloqueio epidural, tendo em vista que o tempo de cirurgia foi, em média, de duas horas e 40 minutos, enquanto o tempo de anestesia foi de três horas e meia. Tal fato revelou-se um achado econômico e vantajoso, já que o animal terá uma recuperação mais rápida, além de haver menor exposição do cão e dos membros da equipe cirúrgica aos gases liberados pelo aparelho de anestesia, que poderia, a longo prazo, gerar alterações de humor, diabetes mellitus, cirrose hepática e mutações genéticas (Harsfield et al., 1996).

\section{CONCLUSÃO}

Conclui-se que a complementação da técnica de anestesia epidural à anestesia geral é eficiente e vantajosa na realização de cirurgias descompressivas lombossacrais, levando ao menor risco anestésico para o animal, menor consumo de anestésico pela equipe cirúrgica e pelo cão, além de um retorno anestésico tranquilo e satisfatório, com menor desconforto doloroso.

\section{REFERÊNCIAS}

ARAUJO, F.P. Avaliação do método semiológico das síndromes neurológicas para o diagnóstico anatômico e etiológico das doenças do sistema nervoso de cães e gatos atendidos no Hospital Veterinário da Universidade Federal Rural de Pernambuco. 35f. Dissertação (Mestrado em Ciências Veterinária) - Universidade Federal Rural de Pernambuco. Recife, 2010.

BECKMANN, D.V. A musculatura epaxial e a fibrose epidural na compressão medular em cães submetidos à laminectomia dorsal modificada. Pesq. Vet. Bras, v.30, p.127-131, 2010.

BLASS, C.E.; SHIRES, P.K. Paralisia respiratória secundária a anestesia epidural em um cão. Cães e Gatos, v.2, p.34-35, 1987.

BIRBACH, D.J.; STEIN, D.J.; Murray, O. et al. Povidine iodine and skin disinfection of epidural anesthesia. Anesthesiol., v.88, p.668-672, 1998.

BOJRAB, M.J. Técnicas atuais em cirurgias de pequenos animais. 3.ed. São Paulo: Roca, 1996. $916 \mathrm{p}$.

BROWN, D.L.; WEDEL, D.J. Anestesia subaracnoidea peridural e caudal. In: MILLER, R.D. Anestesia. 3.ed. São Paulo: Artes Medicas, 1993. p.1377-1405. 
CARIOU, M.P.; STÖRK, C.K.; PETITE, A.F.; RAYWARD, R.M. Cauda equina syndrome treated by lumbosacral stabilization in a cat. Vet. Comp. Orthopedics Traumatol., v.21, p.462-466, 2008.

CARVALHO, Y.K. DE; LUNA, S.P.L Anestesia e analgesia por via epidural em cãesatualização farmacológica para uma técnica tradicional. Clin. Vet., v.70, p.68-76, 2007.

COLLINS, V.J. General and regional anesthesia. 3.ed. Philadelphia: Lea \& Febiger, 1993. 30p.

DOBROMYLSKYJ, P.; FLECKNELL, B.D.; LASCELLES, P.J. et al. Pain assessment. In: FLECKNELL, P.A.; WATERMAN-PEARSON, A. Pain management in animals. London: WB Saunders, 2001. cap. 4, p.53-79.

FANTONI, D.T.; CORTOPASSI, S.R. Anestesia em cães e gatos. 2.ed. São Paulo: ROCA, 2010. v.1, 620p.

FANTONI, D.T.; MASTROCINQUE, S. Fisiopatologia e controle da dor aguda. In: FANTONI, D.T.; CORTOPASSI, S.R.G. Anestesia em cães e gatos. São Paulo: Roca, 2010. cap. 35, p.521-544.

HARSFIELD, S.; McGRATH, C.; GAYNOR, J. et al. Commentary and recommendations on control of waste anesthetic gases in the workplace. J. Am. Vet. Med. Associat., v.209, p.75-77, 1996.

HEBL, J.R.; HORLOCKER, T.T.; SCHROEDER, D.R. Neuraxial anesthesia and analgesia in patients with preexisting central nervous system disorders. Anesth. Analg., v.103, p.223-228, 2006.

INTELIZANO, T.R.; FANTONI, D.T.; CORTOPASSI, S.R.G. Técnicas de anestesia local. In: FANTONI, D.T.; CORTOPASSI, S.R.G. Anestesia em cães e gatos. São Paulo: Roca, cap.19. 2002. p.199-208.

KATZ, J. Pre-emptive analgesia: importance of timing. Canadian J. Anaesthesia, v.48, p.105114, 2001.

LAGEDO, C.M.G.; TUDURY, E.A.; FARIA, M.L.E. Automutilação devido à compressão da cauda equina em três cães e um gato. Cienc. Rural, v.29, p.71-74, 1999.
LAURETTI, G.R.; TREVELLIN, W.; MATTOS, A.L. et al. Avaliação do efeito antinociceptivo do fentanil transdérmico no controle da dor lombar pós-operatória. Coluna/Columna, v.8, p.412-416, 2009.

MASSONE, F. Anestesia veterinária: farmacologia e técnicas. 4.ed. Rio de Janeiro: Guanabara Koogan, 2003. p.225.

McMURPHY, R.M. Postoperative epidural analgesia. Vet. Clin. North Am.: Small Anim. Pract., v.23, p.703-716, 1993.

MICH, P.M.; HELLYER, P.W. Objective, Categorical Methods for Assessing Pain and Analgesia. In: GAYNOR, JS; MUIR, W.W. III, $3^{\circ} \mathrm{ed}$. Handbook of veterinary pain management. St Louis: Mosby, p.78-107, 2008.

NOGUEIRA, L.C.; CORTOPASSI, S.R.G; SOUZA, M.S.B.; INTERLIZANO, T.R. Efeitos do jejum alimentar pré-cirúrgico sobre a glicemia e o período de recuperação anestésica em cães . Braz. J. Vet. Res. Anim. Sci., v.40, p.20-25, 2003.

NUNES, N.; COSTA J.L.O.; NOBREGA NETO, P.I. Efeitos cardio-respiratórios da anestesia epidural em cães não pré-medicados. Cienc. Rural, v.23, p.305-309, 1993.

OLIVEIRA, C.M.B.; SAKATA, R.K.; ISSY, A.M.; GARCIA, J.B.S. Cetamina e analgesia preempitiva. Rev. Bras. Anestesiol., v.54, p.739$752,2004$.

PALMER, R.H.; CHAMBERS, J.N. Canine lumbosacral diseases. Part II. Definitive diagnosis, tratament, and prognosis. The Compendium- Small Animal, v.13, p.213-221, 1991.

PASCOE, P.J. Drugs in the epidural space. In: INTERNATIONAL CONGRESS OF VETERINARY ANAESTHESIOLOGY, 60. 1997, Proceedings... Thessaloniki. p.53-61, 1997.

PINHEIRO, M.R.S.; CECARRELI, M.J.N.B.; CASTRO, L.F.L. et al. Bloqueio do plexo braquial pela via interescalênica em crianças. Rev. Bras. Anestesiol., v.36, p.285-288, 1996.

POLIS, I.; GASTHUYS, F.; VAN HAM, L.; LAEVENS, H. Recovery times and evaluation of clinical hemodynamic parameters or sevoflurane, isoflurane and halothane in mongrel dogs. $J$. Vet. Med. Anim. Physiol., Pathology, Clinical Medicine, v.48, p.401-411, 2001. 
RISIO, L. DE; SHARP, N.J.; OLBY, N.J. et al. Degenerative lumbossacral stenosis. Common neurol. problems, v.30, p.111-132, 2000.

SEBEL, P.S.; LOWDON, J.D. Propofol: a new intravenous anesthetic. Anesthesiol., v.71, p.260277,1989

SEIM III, H.B. Cirurgia da espinha toracolombar. In: FOSSUM, T.W. Cirurgia de pequenos animais. 2. ed. São Paulo: Roca, 2005, cap. 40, p.1259-1291.

SHARP, N.J.H.; WHEELER, S.J. Small animal spinal disorders. Diagnosis and surgery. 2.ed. Philadelphia: Elsevier Mosby, 2005. 380p.

SJOSTROM, L. Distúrbios lombossacrais. In: Manual de cirurgia de pequenos animais. 3. ed. v.1, Manole: São Paulo, 2007. p.1227-1237.
SKARDA, R.T. Local and regional anesthesic and analgesia techniques: dogs. In: THURMON, J.C; TRANQUILLI, W.J; BENSON, G.J. Lumb \& Jones Veterinary Anesthesia. 3.ed. Baltimore: Williams and Wilkins, 1996. p.426-447.

TARVIN, G.; PRATA, R.G. Lumbosacral stenosis in dogs. J. Amer. Vet. Med. Associat., v.177, p.154-159, 1980.

THUMON, J.C.; TRANQUILLI, W.J.; BENSON, G.J. Lumb \& Jones veterinary anesthesia. In:_ Preanesthetics and anesthetic adjuncts. 3.ed. Baltimore : Williams \&Wilkins, Cap.8, 1996. p.183-209.

WILDER-SMITH, O.H.G.; RAVUSSIN, P.A.; DECOSTERD, L.A. Midazolam premedication reduces propofol dose requirements for multiple anesthetic endpoints. Can Journal oh Anesthesia, v.48, p.439-445, 2001. 